\title{
La asimetría y el grado de optimización del árbol bronquial en Rattus norvegicus y Oryctolagus cuniculus
}

\author{
The asymmetry and the optimization degree of the airway of Rattus norvegicus and \\ Oryctolagus cuniculus
}

\section{MAURICIO CANALS ${ }^{1}$, CRISTIAN ATALA ${ }^{1}$, RICARDO OLIVARES ${ }^{2}$, F. FERNANDO NOVOA ${ }^{1} \&$ MARIO ROSENMANN $^{1}$}

\author{
${ }^{1}$ Departamento de Ciencias Ecológicas, Facultad de Ciencias, Universidad de Chile, Casilla 653, \\ Santiago, Chile; e-mail: mcanals@abello.dic.uchile.cl \\ ${ }^{2}$ Departamento de Ciencias Biológicas, Facultad de Ciencias Veterinarias, Universidad de Chile, \\ Casilla 2, Correo 15, La Granja, Santiago, Chile
}

\begin{abstract}
RESUMEN
La vía aérea ha sido propuesta como modelo de diseño óptimo desde una perspectiva física. Su diseño se ha asociado con un adecuado flujo de gases a los alvéolos, una mínima producción de entropía y un mínimo costo en materia y energía. Se ha propuesto un decrecimiento exponencial del diámetro de los bronquios $\left(\mathrm{d}_{\mathrm{G}}\right)$ en función de la generación: $\mathrm{d}_{\mathrm{G}}=\mathrm{do} \cdot 2^{\mathrm{G} / 3}$, asociado a una mínima producción de entropía. También se ha propuesto un modelo de renormalización: $\mathrm{d}_{\mathrm{G}}=\mathrm{A}_{\mathrm{n}} \cdot \mathrm{G}^{-\mathrm{u}}$ donde $\mathrm{u}$ es un exponente y $\mathrm{A}_{\mathrm{n}}$ una función que introduce desviaciones periódicas en la escala, es decir más de una escala, evitando la propagación distal de errores aleatorios en el calibre de un bronquio. Sin embargo, este último resultado podría ser consecuencia en árboles asimétricos de la relación entre el diámetro y el orden del bronquio y no de la generación. En este trabajo estudiamos la asimetría y el decrecimiento del diámetro bronquial en dos especies. Se utiliza el modelo de Zamir como un sistema externo de medida de la optimización. Encontramos una clara asimetría del árbol bronquial. Comprobamos que la relación exponencial diámetro-orden es siempre muy buena $\left(\mathrm{R}^{2} \approx 0,8\right)$ y que en cambio la relación exponencial diámetro-generación es menos clara $\left(\mathrm{R}^{2}<0,6\right)$. La supuesta modulación armónica desaparece al considerar el orden y no la generación. Se determina un alto grado de optimización de la estructura en las dos especies.
\end{abstract}

Palabras clave: optimización, bronquios, asimetría, modelos.

\begin{abstract}
From a physical perspective, the air way has been proposed as a model of optimal design. Its design has been associated with a optimal gases flow to the alveoli, a minimum entropy production and minimal costs of mass and energy. The decrease of the bronchial diameter $\left(\mathrm{d}_{\mathrm{G}}\right)$ along the airway has been modeled by (i) an exponential decay of the bronchial diameter $\left(d_{G}\right)$ as function of its generation: $d_{G}=d o \cdot 2^{-G / 3}$, associated to a minimum entropy production, and (ii) a renormalization model: $d_{G}=A_{n} \cdot G^{-u}$ where $u$ is an exponent and $A_{n}$ an harmonic function which introduces periodic variations in the scale, buffering the propagation of stochastic errors in the bronchial diameter. However, that the last result in asymmetric trees may be a consequence of a real relationship between diameter and order more than a relationship diameter-generation. In this work we explore this hypothesis in two species. We also use the Zamir model for vascular trees as an out method to explore the optimality degree of the bronchial ramification. We found a clear asymmetry of the analyzed bronchial trees. We shown that the relationship diameter-order is always very good $\left(\mathrm{R}^{2} \approx\right.$ $0,8)$. In contrast, the determination coefficients for the relationship diameter-generation were lower $\left(R^{2}<0,6\right)$. The proposed harmonic modulation vanishes when changing generation by order. There were a high optimality degree of the bronchial junctions.
\end{abstract}

Key words: optimality, airway, asymmetry, models.

\section{INTRODUCCIÓN}

Las especies animales muestran una gran diversidad en su diseño estructural a consecuencia de su adaptación a diferentes condiciones de vida. Esta observación sugiere preguntas como ¿está su diseño optimizado? o ¿los animales presentan un diseño económico con no mas estructura que la necesaria para llevar a cabo su función?. Un ejemplo de diseño económico es la estructura fina del hueso, ajustada cualitativa y cuantitativamente a las tensiones mecánicas que debe soportar (Dullemeijer 1998, Weibel et al. 1998). Otro ejemplo lo constituye la variación del diámetro de las 
ramas vasculares donde se minimiza la masa de sangre, la energía de transporte y la resistencia al flujo (Zamir 1978, 1986, Weibel et al. 1998).

Una hipótesis que enmarca el diseño económico es la simorfosis, que en su aspecto esencial señala que si el apareamiento estructura-función (morfogénesis regulada) es acoplado a la estricta economía de energía y materiales el resultado sería un diseño de órganos optimizados para la función que realizan (Weibel et al. 1991, 1992). Sin embargo, esta posición ha despertado algún escepticismo (Garland \& Huey 1987, Dudley \& Ganz 1991, Jones 1998). Desde una perspectiva evolutiva puede preguntarse si la selección natural conduce a un diseño óptimo o simplemente adecuado, o si el proceso de optimización, si lo hay, opera a nivel de individuo o de órganos o niveles inferiores. Por otra parte si existe un diseño óptimo, ¿lo es en cuanto a su función? ¿en cuanto al gasto de energía y materiales? ¿mínima producción de entropía? ¿máximo aprovechamiento del espacio? Todos estos criterios ¿conducen a un mismo diseño? o los animales deben privilegiar un aspecto determinando costos en otro?

El sistema respiratorio ha sido usado largamente como modelo de estudio del acoplamiento entre la estructura-función y la economía en energía y materiales.

En una serie de estudios incluyendo gran variedad de especies, Weibel et al. (1992) encontraron que el volumen mitocondrial, el gasto cardiaco máximo y el volumen capilar varían alométricamente con la masa corporal pero con el mismo exponente que el consumo de oxígeno máximo $\left(\mathrm{V}_{\mathrm{O} \text { max }}\right)$ de tal manera que la mitocondria tiene la misma tasa de fosforilación oxidativa en todos los mamíferos y lo mismo ocurre con el volumen capilar y el gasto cardíaco, lo que es un fuerte apoyo a la simmorfosis: si las capacidades máximas son fijas implica que la capacidad total está determinada por propiedades de diseño estructural y entonces ajustes de la capacidad funcional por cambios en las demandas requieren procesos morfogenéticos (Weibel et al. 1991, 1992). Sin embargo los mismos autores encontraron que la capacidad de difusión pulmonar estaba sólo parcialmente ajustada a $\mathrm{V}_{\mathrm{O} \text { max }}$, y entonces algunas especies tienen un exceso de capacidad de difusión mientras que otras no, especialmente las pequeñas o entrenadas físicamente.

En cuanto al diseño del árbol bronquial, la vía aérea ha sido propuesta como modelo de diseño óptimo desde una perspectiva física (Rhorer 1915, Weibel \& Gómez 1962, Wilson 1967). Su diseño se ha asociado con un adecuado flujo de gases a los alvéolos (Rhorer 1915), con una mínima producción de entropía (Wilson 1967) y con un mínimo costo en materia y energía (Weibel et al. 1998 ).

Weibel y Gómez (1962) encontraron un decrecimiento exponencial del diámetro de los bronquios $\left(\mathrm{d}_{\mathrm{G}}\right)$ en función de la generación $(\mathrm{G})$ :

$$
\mathrm{d}_{\mathrm{G}}=\mathrm{do} \cdot 2^{-\mathrm{G} / 3}
$$

Posteriormente, Wilson (1967) estudiando la producción total de entropía (S) a nivel del sistema respiratorio $\mathrm{S}=\mathrm{H} \mathrm{V}_{\mathrm{O} 2} / \mathrm{T}$, donde $\mathrm{H}$ es la energía disipada por $\mathrm{ml}$ de $\mathrm{O}_{2}$ consumido y $\mathrm{T}$ la temperatura, demostró matemáticamente que este decrecimiento exponencial con la generación y su exponente estaban asociados a una mínima producción de entropía $\left(\delta \mathrm{S} / \delta \mathrm{d}_{\mathrm{G}}=0\right)$ estableciendo así formalmente la relación entre decrecimiento exponencial del diámetro y producción mínima de entropía. Weibel \& Gómez (1962) observaron que el comportamiento de la reducción del diámetro bronquial se apartaba levemente de su modelo exponencial a partir de la generación décima. Ellos explicaron este hecho por un cambio en los principios físicos dominantes en el transporte de los gases, desde una zona caracterizada por el movimiento de los gases en el interior de la vía de conducción a una zona donde predomina el transporte por difusión.

Estudios posteriores, aunque no han contradicho lo anterior, han demostrado una geometría fractal del árbol bronquial y otras estructuras pulmonares (Rigaut 1984, Mandelbrot 1989, Glenny \& Robertson 1990, Horsfield 1990, Nelson et al. 1990, Canals et al. 1998, 2000). La geometría fractal del decrecimiento del diámetro del árbol bronquial se ha caracterizado por una ley de potencias (modelo de renormalización)

$$
\mathrm{d}_{\mathrm{G}}=\mathrm{A}_{\mathrm{n}} \cdot \mathrm{G}^{-\mathrm{u}}
$$

donde $\mathrm{G}$ es la generación, $\mathrm{u}$ un exponente y $\mathrm{A}_{\mathrm{n}}$ una función que introduce desviaciones periódicas en la escala, y no por un decrecimiento exponencial. West et al. (1986) propusieron que la desviación desde un modelo exponencial no era explicada por una zona de transición. Ellos propusieron que su modelo de renormalización indicaba que en la reducción del calibre de la vía aérea de una generación a otra interviene más de una escala. Mas bien ocurriría una oscilación armónica en torno a una escala que podría evitar la propagación distal de errores aleatorios en el calibre de un bronquio (McNamee 1991). En el hombre, este modelo predice adecuadamente el diámetro de la vía aérea a través de 20 generaciones de bronquios y representa adecuadamente la vía aérea de perros, ratas y hámsters (Nelson et al. 1990). Sin embra- 
go, Horstfield (1990) muestra en forma teórica que el resultado de West et al. (1986) podría ser por un lado una consecuencia de la asimetría del árbol bronquial y por otra parte de la relación exponencial que existe entre el diámetro y el orden del bronquio y no de la generación. Así, los hallazgos de West et al. (1986) no serían incompatibles con los de Weibel \& Gómez (1962).

La diferencia en el sistema de numeración está en que la generación se numera de proximal a distal y el orden a la inversa, aumentando en 1 el orden cada vez que recibe una rama. Si el árbol es simétrico el modelo es insensible al sistema de numeración, pero si es asimétrico cambia el ajuste del modelo y sus parámetros (Horstfield 1990).

El problema se hace mayor cuando consideramos la siguiente pregunta: ¿ cambia la generación $\mathrm{u}$ orden de una rama bronquial cuando da origen o recibe otra de un diámetro muy inferior? Numerando las generaciones y usando el sistema de Horstfield (1990) para los órdenes esto debiera ser así; sin embargo Strahler (1957) propuso una numeración diferente a partir de la numeración de los afluentes de los ríos que se utiliza mucho en el estudio de árboles vasculares (Strahler 1957, Shreiner et al. 1996). En este sistema, se numeran con 0 las ramas terminales y las ramas sólo cam- bian de orden cuando reciben una de igual orden igual (Fig. 1).

Este problema de la asimetría del árbol bronquial y su sensibilidad al sistema de numeración es de gran importancia en el estudio del grado de optimización de las vías de conducción desde una perspectiva física por cuanto la relación entre diámetro y el orden del bronquio establece una relación con el flujo (q) que transporta, a través $\mathrm{de} \mathrm{q}=\mathrm{k} \cdot \mathrm{d}^{\mathrm{x}}$, con $\mathrm{k}$ una constante, $\mathrm{d}$ el diámetro $\mathrm{y}$ $\mathrm{x}$ un exponente (i.e., $\mathrm{q}=\mathrm{k} \cdot \mathrm{d}^{3}$ ) (Horstfield et al. 1976, Zamir 1978, Sherman 1981). Así, de existir un flujo óptimo a nivel de la vía aérea, basado en la relación flujo diámetro y de éste con el orden del bronquio, se ha propuesto para árboles dicotómicos que la razón de incremento exponencial del diámetro en función del orden $\left(\mathrm{Rd}^{*}\right)$ sigue a la raíz cúbica de la razon de incremento exponencial del número de ramas $(\mathrm{Rb})$ : $\mathrm{Rd}^{*}=\mathrm{Rb}^{1 / 3}$ (Horstfield et al. 1976, Horstfield 1977, Horstfield \& Thurlbeck 1981). Una relación similar usa Wilson (1967) para proponer la relación entre el modelo exponencial y la producción mínima de entropía, pero basado en la generación.

Zamir (1976, 1978, 1986) y Zamir \& Bigelow (1984) estudiaron los principios de optimización
(A)
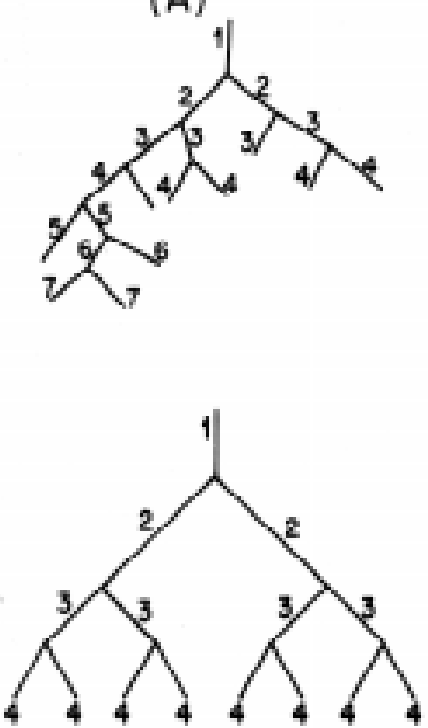

(B)
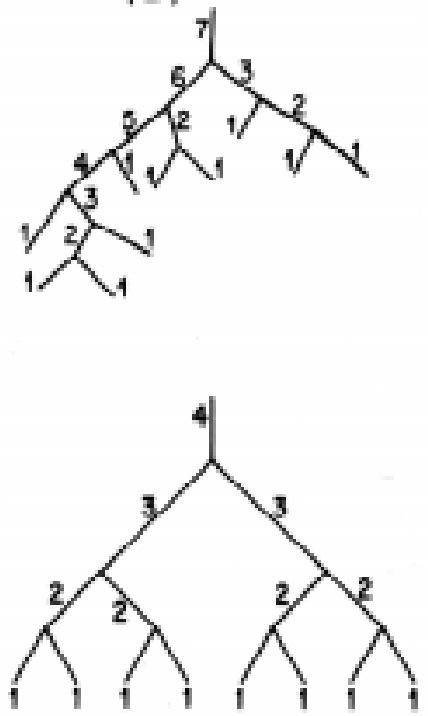
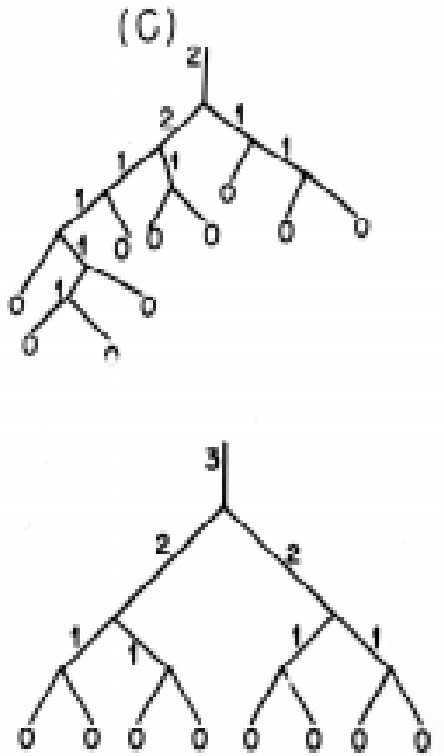

Fig. 1: Numeración de generaciones y órdenes de las ramas del árbol bronquial en árboles asimétricos (arriba; $\Delta=3$, ver texto) y simétricos (abajo): (A) generaciones, G; (B) orden de Horstfield, H; (C) orden de Strahler, S. Se puede observar que en árboles simétricos los sistemas de numeración son intercambiables: $\mathrm{S}=\mathrm{H}-1$ y $\mathrm{H}=\mathrm{G}_{\max }-\mathrm{G}+1$. Sin embargo esto no ocurre en árboles asimétricos, donde además bronquios terminales son atribuidos a generaciones diferentes (entre cuatro y siete en la figura).

Generations and orders in branches of asymmetrical (up; $\Delta=3$, see text) and symmetrical air way (down): (A) generations, G; (B) Horstfield order, H; (C) Strahler order, S. In the symmetrical trees the numeration systems are interchangeable: $\mathrm{S}=$ $\mathrm{H}-1$ and $\mathrm{H}=\mathrm{G}_{\max }-\mathrm{G}+1$. However, this is not true in asymmetrical trees where furthermore terminal branches are attributed to different generations (four to seven in the figure). 
de árboles vasculares utilizando otro enfoque, independiente de la asimetría y del sistema de numeración. Zamir propuso la bifurcación como la unidad de estudio y cuatro hipótesis: una bifurcación se encuentra en estado óptimo si es mínimo (i) el área del lumen, (ii) el volumen total de las ramificaciones, (iii) la potencia requerida para el movimiento e (iv) el roce en las paredes. El modelo de Zamir proporciona los ángulos óptimos de bifurcación $\left(\theta_{\mathrm{i} 0}\right)$ asociados a determinados índices de bifurcación $(\alpha)$ que corresponden a la razones entre los diámetros de los vasos hijos: $\alpha=\mathrm{d}_{2} / \mathrm{d}_{1}, \mathrm{~d}_{2}$ el diámetro del bronquio menor (Fig. 2). También demostró que si se considera la relación entre flujo (q) y radio (r),

$$
q=c \cdot r^{1 / 3}(c, \text { una constante }),
$$

los ángulos óptimos para mínima potencia y mínimo volumen coinciden y lo mismo ocurre para los ángulos óptimos para mínima área y roce (Zamir 1978), así:

$$
\begin{aligned}
& \cos \mathrm{q}_{10}=\left(\left(1+\mathrm{a}^{3}\right)^{4 / 3}+1-\mathrm{a}^{4}\right) /\left(2\left(1+\mathrm{a}^{3}\right)^{2 / 3}\right) \\
& \cos \mathrm{q}_{20}=\left(\left(1+\mathrm{a}^{3}\right)^{4 / 3}+\mathrm{a}^{4}-1\right) /\left(2 \mathrm{a}^{2}\left(1+\mathrm{a}^{3}\right)^{2 / 3}\right) \\
& \text { para mínimo volumen y potencia y } \\
& \cos \mathrm{q}_{10}=\left(\left(1+\mathrm{a}^{3}\right)^{2 / 3}+1-\mathrm{a}^{2}\right) /\left(2\left(1+\mathrm{a}^{3}\right)^{1 / 3}\right) \\
& \cos \mathrm{q}_{20}=\left(\left(1+\mathrm{a}^{3}\right)^{2 / 3}+\mathrm{a}^{2}-1\right) /\left(2 \mathrm{a}\left(1+\mathrm{a}^{3}\right)^{1 / 3}\right) \\
& \text { para mínimo roce y área. }
\end{aligned}
$$

Zamir \& Bigelow (1984) desarrollaron un método de representación del óptimo en un gráfico rectangular llamado "junction box" (Fig. 3). En este gráfico el óptimo es representado por un punto $\left(\mathrm{x}_{0}, \mathrm{y}_{0}\right)$ determinado por los ángulos $\theta_{\mathrm{i}} \mathrm{y}$ una determinada bifurcación observada por otro punto $(\mathrm{x}, \mathrm{y})$, de manera que la distancia al óptimo

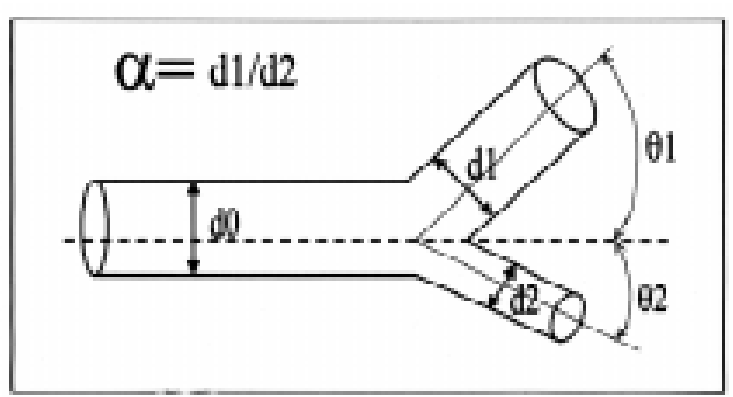

Fig. 2: La unidad estructural básica del árbol bronquial: la bifurcación. En una bifurcación un bronquio parental de diámetro do da origen a dos ramas de diámetros $\mathrm{d}_{1} \mathrm{y} \mathrm{d}_{2}$ (el más fino) con ángulos $\theta_{1}$ y $\theta_{2}$.

The basic structural unit of the bronchial tree: the junction. At a bifurcation site a parental bronchi of diameter $d_{0}$ splits into two branches of diameters $d_{1}$ and $d_{2}$ (the smallest) with branching angles $\theta_{1}$ and $\theta_{2}$.

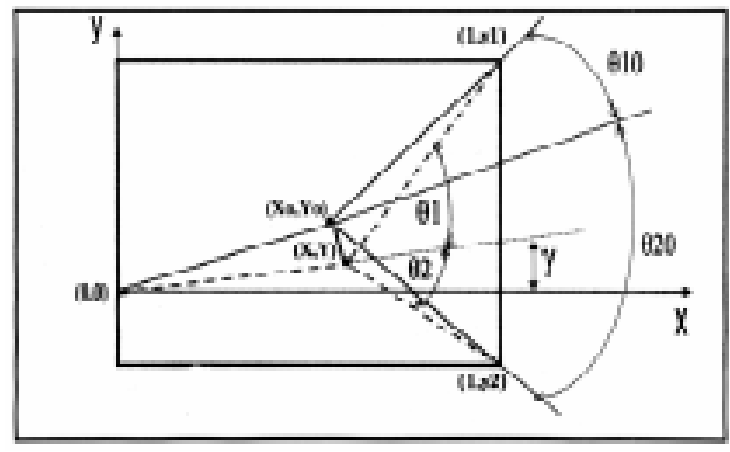

Fig. 3: "Junction box" de Zamir \& Bigelow (1984). La bifurcación óptima es enmarcada en una caja en la cual las tres ramas de la bifurcación tienen la misma longitud. Después en la misma caja se ajusta una bifurcación observada. la distancia entre el punto observado $(\mathrm{x}, \mathrm{y})$ y el punto óptimo (xo, yo) determina el costo de alejamiento del óptimo.

The "junction box" of Zamir \& Bigelow (1984). The optimum bifurcation is enclosed in a box such that the three branches forming the bifurcation have equal lengths. Then, a measured bifurcation is fitted in the same box. The distance to which the measured point $(\mathrm{x}, \mathrm{y})$ is away from the optimum point (xo, yo) determines the cost of departure from optimality.

representa cuan lejos se encuentra de éste (Zamir 1978).

El objetivo de este trabajo es estudiar la influencia de la asimetría y de los sistemas de numeración de órdenes y generaciones en el estudio de la optimización física del árbol bronquial desde las perspectivas del decrecimiento exponencial del diámetro (modelo de entropía mínima) y del modelo independiente de Zamir (modelo de optimización de área, volumen, roce y potencia).

\section{MATERIALES Y MÉTODOS}

Trabajamos con dos ratas blancas adultas (R1 y R2) (Rattus norvegicus: 338,4 y $323,7 \mathrm{~g}$ ) y dos conejos (C1 y C2) (Oryctolagus cuniculus: $3.167,4$ y $2.856,0 \mathrm{~g}$ ). Los animales fueron sacrificados, realizándoseles inmediatamente una broncografía con un cateter plástico en la tráquea. Se inyectaron entre 1,5 y $2 \mathrm{ml}$ de sulfato de bario diluido llenando hasta la vía aérea fina por desplazamiento con aire. El procedimiento fue realizado con visualización radioscópica en el servicio de Radiología del Hospital Del Salvador. Se tomaron radiografías a $1 \mathrm{~m}$, con $100 \mathrm{~mA}, 0,04 \mathrm{~s}$ y $34 \mathrm{kV}$. Las broncografías fueron digitalizadas y estudiadas con el programa Scion Image. Se determinó en cada árbol bronquial la generación (G) y el 
orden de cada bronquio con los sistemas de Horstfield (H) y de Strahler (S), y se midieron sus diámetros. Se compararon las pendientes de decaimiento del diámetro a lo largo de la vía aérea con ANCOVA anidado. Se contó el número de ramas correspondientes a cada orden de Horstfield $\left(\mathrm{N}_{\mathrm{H}}\right)$. Se estudió la asimetría mediante la diferencia de órdenes de Horstfield de los bronquios "hijos" en cada bifurcación (coeficiente de asimetría: $\Delta$; Horstfield 1990). Este coeficiente es igual a cero en árboles simétricos $\mathrm{y} \geq 1$ en árboles asimétricos.

Se escogieron al azar entre seis y siete bifurcaciones en cada árbol bronquial midiendo diámetros y ángulos estimando los ángulos óptimos para cada razón de diámetros y finalmente las distancias al óptimo esperado según la metodología de Zamir (1978) y Zamir \& Bigelow (1984) construyendo la "junction box" (Fig. 3) y estimando la función de costo:

$$
\begin{aligned}
& \mathrm{H}=\left(\mathrm{x}^{2}+\mathrm{y}^{2}\right)^{1 / 2}+\left[(1-\mathrm{x})^{2}+(\mathrm{y} 1-\mathrm{y})^{2}\right]^{1 / 2} \cdot \mathrm{h}_{1}+ \\
& {\left[(1-\mathrm{x})^{2}+(\mathrm{y} 2-\mathrm{y})^{2}\right]^{1 / 2} \cdot \mathrm{h}_{2}}
\end{aligned}
$$

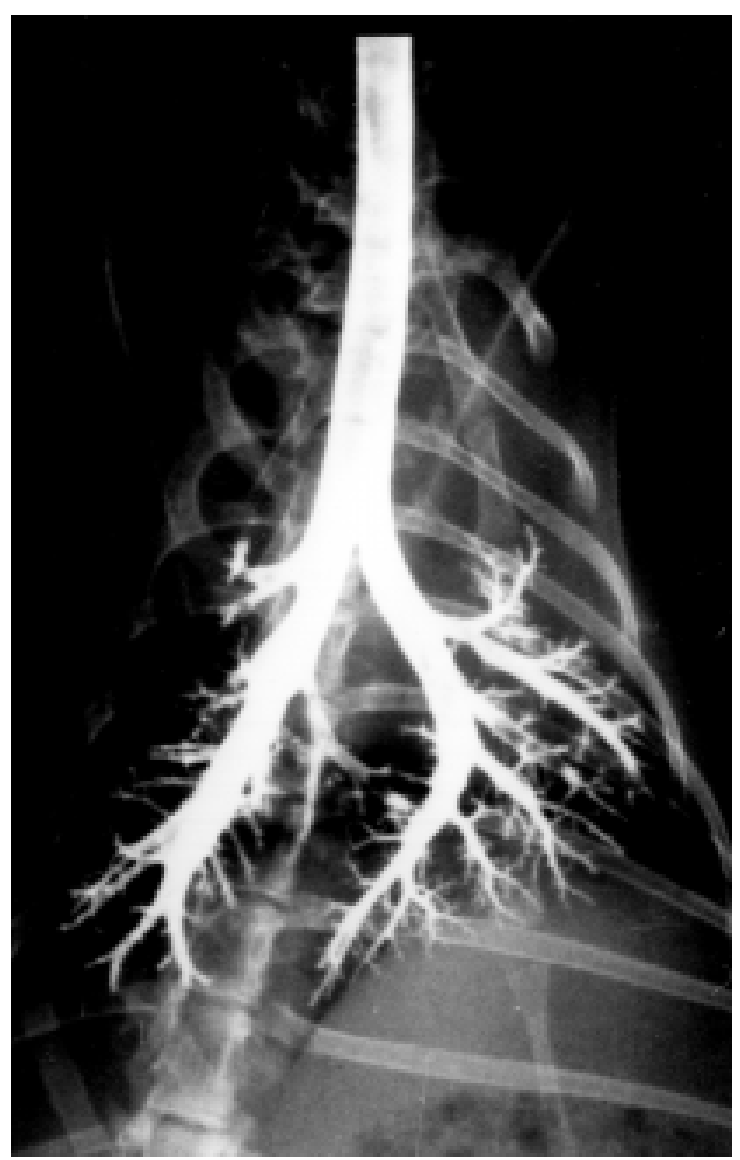

Fig. 4: Broncografía de conejo (C1).

Bronchograhy of a rabbit (C1). donde $h_{1}=\left(1+a^{3}\right)^{-2 / 3}, h_{2=} a^{2} \cdot\left(1+a^{3}\right)^{-2 / 3}$ para mínimos volumen y potencia, y $h_{1}=\left(1+a^{3}\right)^{-1 / 3}, h_{2}=$ $\mathrm{a} \cdot\left(1+\mathrm{a}^{3}\right)^{-1 / 3}$ para mínimas superficie y roce (Zamir 1978).

Se compararon ángulos y distancias obtenidas con ANOVA anidado de una y dos vías respectivamente.

Para cada árbol bronquial se estimó su coeficiente de asimetría $(\Delta)$ y los siguientes parámetros: razón de incremento exponencial del número de ramas por orden de Horstfield: $\mathrm{Rb}=$ antilog $\mid$ pendiente | en el gráfico $\log \left(\mathrm{N}_{\mathrm{H}}\right)$ versus $\mathrm{H}$; razón de incremento exponencial del diámetro por orden de Horstfield: $\mathrm{Rd}=$ antilog $\mid$ pendiente $\mid$ en el

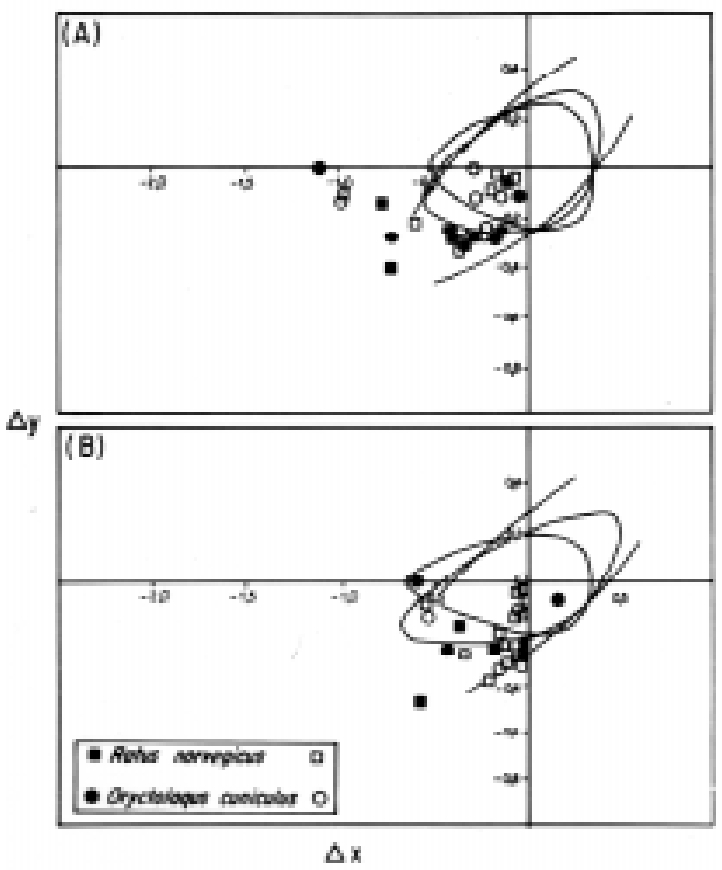

Fig. 7: Datos de la ramificación bronquial de dos conejos (C1 y C2) y dos ratas (R1 y R2) transformadas en su equivalencia en costo (distancias al óptimo) graficadas para la interpretación de su grado de optimización bajo los criterios de (A) mínimos superficie y roce y (B) mínimos volumen y potencia. Las líneas de cortorno representan las isolíneas de el $5 \%$ de variación alrededor del punto óptimo para índices de bifurcación $\alpha=0,1$; 0,6 y 1,0 (penalización del costo; de Zamir \& Bigelow 1984).

Bronchi branching data of two rabbits ( $\mathrm{C} 1$ and $\mathrm{C} 2)$ and two rats (R1 and R2) transformed into their cost-equivalent (distances to the optimum), plotted for interpretation of their degree of optimality with respect to (A) minimum drag and lumen surface and to (B) minimum pumping power and lumen volume. The contours shown in the plot represent the isolines of the five percent variation around the minimum cost for three bifurcation index $\alpha=0.1,0.6$ and 1.0 ("cost penalties"; from Zamir \& Bigelow 1984). 

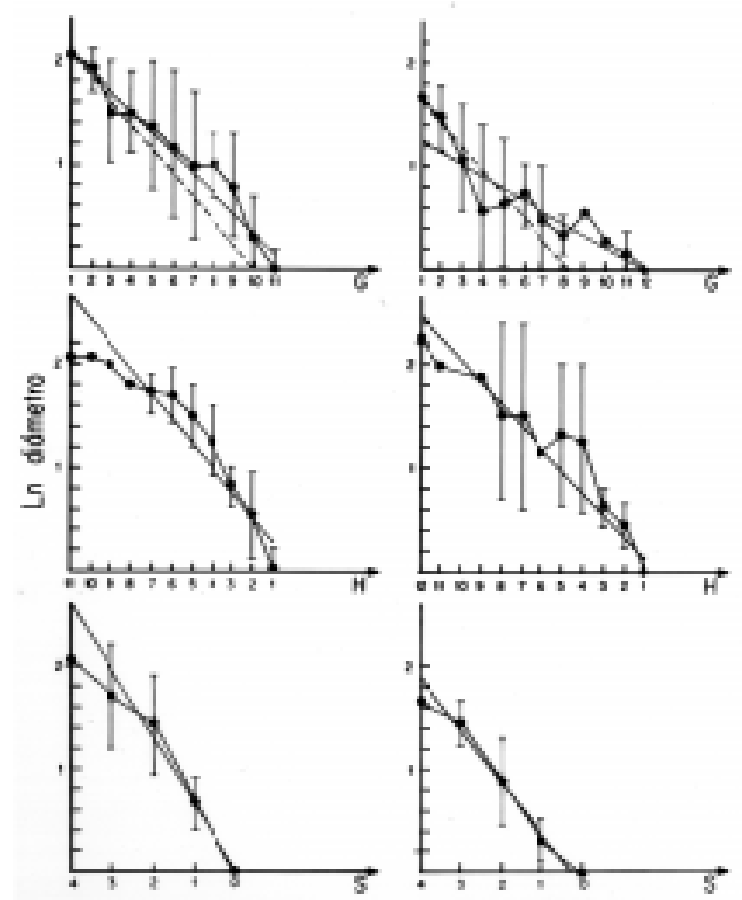

Fig. 5: Variación del diámetro bronquial en función de la generación (G), el orden de Horstfield (H) y el orden de Strahler (S) para dos conejos ( $\mathrm{C} 1$ a la izquierda y $\mathrm{C} 2$ a la derecha). La línea segmentada en los gráficos superiores representa la variación esperada según el modelo de Weibel \& Gómez (1962).

Bronchial diameter variation as function of the generation $(G)$, Horstfield order $(H)$ and Strahler order (S) in two rabbits (C1 left side and $\mathrm{C} 2$ right side). The dashed line represents the expected decay of the diameter following the generation from the Weibel \& Gómez (1962)'s model.
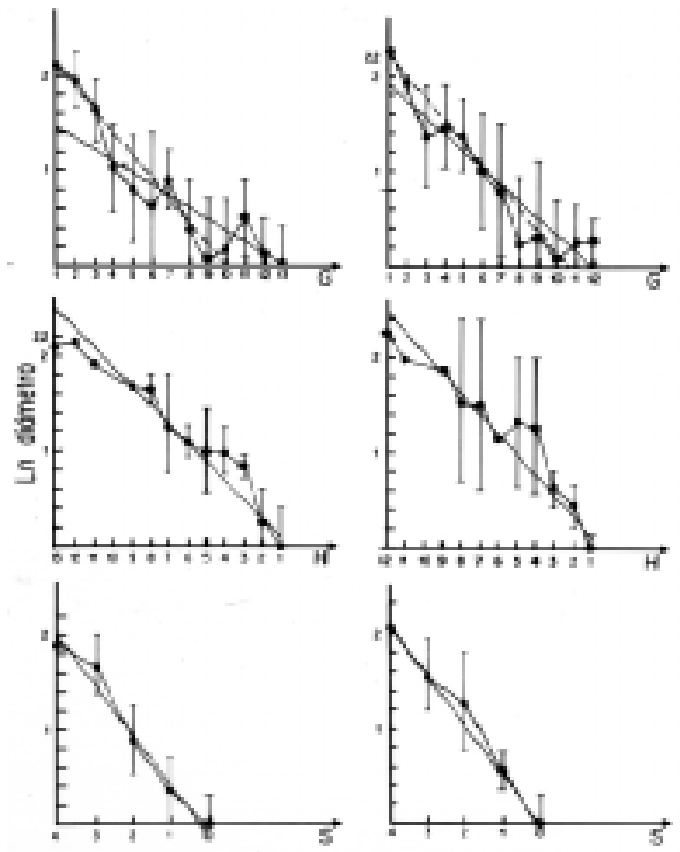

Fig. 6: Variación del diámetro bronquial en función de la generación $(\mathrm{G})$, el orden de Horstfield (H) y el orden de Strahler (S) para dos ratas (R1 a la izquierda y R2 a la derecha). La línea segmentada en los gráficos superiores representa la variación esperada según el modelo de Weibel \& Gómez (1962).

Bronchial diameter variation as function of the generation $(G)$, Horstfield order $(H)$ and Strahler order (S) in two rats (R1 left side and R2 right side). The dashed line represents the expected decay of the diameter following the generation from the Weibel \& Gómez (1962)'s model. 
gráfico $\log (\mathrm{d})$ versus $\mathrm{H}$; razón de incremento exponencial del diámetro por orden de Strahler: $\mathrm{Rd}(\mathrm{s})=$ antilog $\mid$ pendiente $\mid$ en el gráfico $\log (\mathrm{d})$ versus $\mathrm{S}$ y razón de decrecimiento exponencial del diámetro por generación $\mathrm{Rd}(\mathrm{g})=$ antilog | pendiente | en el gráfico log (d) vs G. Se compara $\operatorname{Rd}(\mathrm{g})$ observado con lo esperado según el modelo de Weibel \& Gómez (1962): $\mathrm{d}_{\mathrm{G}}=$ do $2^{-\mathrm{G} / 3}$, $\mathrm{d}_{\mathrm{G}}=$ diámetro del bronquio de la generación $\mathrm{G}$, este es equivalente a $\log \mathrm{d}=\log \mathrm{do}-0,1003433$ $\mathrm{G}$, esperando que $\mathrm{Rd}(\mathrm{g})=\operatorname{antilog}(0,1003433)=$ 1,2599. Se compara además éste parámetro $(\operatorname{Rd}(\mathrm{g}))$ con lo esperado para árboles asimétricos con un coeficiente de asimetría $\Delta=3$ (valores de Horstfield 1990). Para las comparaciones se utilizó una prueba t de Student para pendientes.

\section{RESULTADOS}

El árbol bronquial de los individuos considerados fue fuertemente asimétrico (Fig. 4). El coeficiente de asimetría fue muy similar al compararlo entre especies e individuos: Conejo 1: $\Delta=3,25 \pm$ 2,1 ; Conejo 2: $\Delta=2,68 \pm 2,0$; Rata $1: \Delta=3,60 \pm$ 2,0 ; Rata $2: \Delta=2,82 \pm 1,5\left(\mathrm{~F}_{1,2}=0,78 ; \mathrm{F}_{2,56}=1,51\right.$; $\mathrm{P}>0,05)$.

El diámetro de la vía aérea decreció en función de la generación y aumentó en función del orden en forma cercanamente exponencial (Tabla 1 y 2 , Fig. 5 y 6). No se detectaron diferencias entre especies ni individuos $\left(\mathrm{F}_{1,151}=0,244 ; \mathrm{P}>0,05\right.$; $\left.\mathrm{F}_{2,135}=1,08 ; \mathrm{P}>0,05\right)$. Sin embargo el ajuste fue

\section{TABLA 1}

Diámetro bronquial promedio (d) y su desviación estándar (s) en dos conejos (C1 y C2), clasificados según generación $(\mathrm{G})$, orden de Horstfield $(\mathrm{H})$ y orden de Strahler (S). Entre paréntesis se consigna el número muestral (n)

Average bronchial diameter (d) and standard deviation (s) in two rabbits (C1 and C2), by generation (G), Horstflied's order (H) and Strahler's order (S). The sample size (n) is showed in brackets

\begin{tabular}{|c|c|c|c|c|c|c|c|c|}
\hline \multirow[b]{2}{*}{$\mathrm{G}(\mathrm{n})$} & \multirow[b]{2}{*}{$\mathrm{d}$} & \multirow[b]{2}{*}{$\mathrm{s}$} & \multicolumn{3}{|c|}{ (C1) } & \multirow[b]{2}{*}{$\mathrm{S}(\mathrm{n})$} & \multirow[b]{2}{*}{$\mathrm{d}$} & \multirow[b]{2}{*}{$\mathrm{s}$} \\
\hline & & & $\mathrm{H}(\mathrm{n})$ & d & $\mathrm{s}$ & & & \\
\hline $1(1)$ & 4,15 & & $1(15)$ & 0,51 & 0,16 & $0(14)$ & 0,50 & 0,15 \\
\hline $2(2)$ & 3,63 & 1,22 & $2(9)$ & 0,71 & 0,28 & $1(10)$ & 0,76 & 0,26 \\
\hline $3(3)$ & 2,71 & 0,89 & $3(4)$ & 1,21 & 0,14 & $2(12)$ & 1,28 & 0,44 \\
\hline $4(4)$ & 1,56 & 0,82 & $4(3)$ & 1,38 & 0,34 & $3(7)$ & 2,81 & 0,95 \\
\hline $5(4)$ & 1,25 & 0,74 & $5(3)$ & 1,50 & 0,70 & $4(2)$ & 3,46 & 0,97 \\
\hline $6(3)$ & 1,21 & 1,05 & $6(2)$ & 1,56 & 0,24 & & & \\
\hline $7(4)$ & 1,30 & 0,45 & $7(3)$ & 1,90 & 0,86 & & & \\
\hline $8(4)$ & 0,87 & 0,58 & $8(2)$ & 2,68 & 0,36 & & & \\
\hline $9(4)$ & 0,65 & 0,50 & $9(1)$ & 2,77 & & & & \\
\hline $10(4)$ & 0,69 & 0,47 & $10(1)$ & 3,01 & & & & \\
\hline $11(4)$ & 0,90 & 0,36 & $11(1)$ & 3,46 & & & & \\
\hline $12(4)$ & 0,61 & 0,22 & $12(1)$ & 4,50 & & & & \\
\hline \multirow[t]{2}{*}{$13(4)$} & 0,52 & 0,20 & $13(1)$ & 4,15 & & & & \\
\hline & \multicolumn{7}{|c|}{ (C2) } & \\
\hline $\mathrm{G}(\mathrm{n})$ & d & $\mathrm{s}$ & $\mathrm{H}(\mathrm{n})$ & $\mathrm{d}$ & $\mathrm{s}$ & $S(n)$ & d & $\mathrm{s}$ \\
\hline $1(1)$ & 4,50 & & $1(12)$ & 0,48 & 0,17 & $0(12)$ & 0,48 & 0,17 \\
\hline $2(2)$ & 3,28 & 0,24 & $2(2)$ & 0,78 & 0,12 & $1(6)$ & 0,87 & 0,15 \\
\hline $3(4)$ & 2,07 & 1,02 & $3(7)$ & 0,91 & 0,19 & $2(13)$ & 1,85 & 0,86 \\
\hline $4(4)$ & 2,16 & 0,80 & $4(2)$ & 1,73 & 0,49 & $3(6)$ & 2,39 & 0,79 \\
\hline $5(4)$ & 1,99 & 0,71 & $5(4)$ & 1,90 & 0,60 & $4(2)$ & 3,81 & 0,97 \\
\hline $6(3)$ & 1,55 & 1,05 & $6(1)$ & 1,55 & & & & \\
\hline 7 (3) & 1,27 & 0,99 & $7(3)$ & 2,31 & 0,80 & & & \\
\hline $8(2)$ & 0,69 & 0,49 & $8(4)$ & 2,38 & 0,90 & & & \\
\hline $9(4)$ & 0,86 & 0,82 & $9(2)$ & 3,12 & 0,01 & & & \\
\hline $10(4)$ & 0,61 & 0,41 & $10(1)$ & 3,38 & & & & \\
\hline $11(4)$ & 0,65 & 0,26 & $11(1)$ & 3,46 & & & & \\
\hline $12(4)$ & 0,65 & 0,17 & $12(1)$ & 4,50 & & & & \\
\hline
\end{tabular}


siempre mejor en la relación entre diámetro y orden de Horstfield o Strahler que en la relación diámetro-generación. Aunque todos los modelos presentaron un adecuado ajuste con coeficientes de determinación altos, el coeficiente de determinación entre diámetro y generación fue siempre el menor en cada individuo (Tabla 3 ).

Los árboles bronquiales fueron aproximadamente dicotómicos con razones $\mathrm{Rb}$ entre 1,32 y 1,44 , lo que es muy similar a lo esperado $(1,38)$ en un árbol bronquial asimétrico en que cada rama da origen a dos (dicotomía), con una asimetría de $\Delta=3$ (Tabla 4): $\mathrm{t}_{11}=0,44, \mathrm{t}_{10}=0,67, \mathrm{t}_{9}=1,11 \mathrm{y} \mathrm{t}_{10}=1,46$ para $\mathrm{C} 1, \mathrm{C} 2, \mathrm{R} 1$ y $\mathrm{R} 2$ respectivamente; $\mathrm{P}>0,05$.

Las razones de incremento exponencial del diámetro en función del orden de Strahler fueron siempre mayores como era de esperar pues este sistema de numeración es mucho mas conservador, ascendiendo de orden sólo cuando una rama recibe otra de orden similar.

El ajuste de el modelo diámetro-generación difirió significativamente de la curva esperada por Weibel \& Gómez (1962) para árboles simétricos en tres de los cuatro casos: $\mathrm{C} 1\left(\mathrm{t}_{44}=4,43 ; \mathrm{P}<0,01\right)$, $\mathrm{C} 2\left(\mathrm{t}_{38}=2,21 ; \mathrm{P}<0,05\right), \mathrm{R} 2\left(\mathrm{t}_{35}=5,07 ; \mathrm{P}<0,01\right)$, $\mathrm{R} 1\left(\mathrm{t}_{36}=1,57 ; \mathrm{P}>0,05\right)$. Al comparar con lo esperado para árboles de asimetría $\Delta=3$, se aprecia un ajuste adecuado en $\mathrm{C} 1$ y R2 $\left(\mathrm{t}_{44}=0,1 ; \mathrm{P}>\right.$ 0,05 y t $\left._{35}=0,2 ; \mathrm{P}>0,05\right)$ pero no en $\mathrm{R} 1$ y C2 $\left(\mathrm{t}_{36}\right.$ $=2,86 ; \mathrm{P}<0,05$ y t$\left._{38}=5,50 ; \mathrm{P}<0,01\right)($ Tabla 4$)$.

Los ángulos de bifurcación fueron similares entre especies e los individuos en las bifurcaciones estudiadas $\left(\mathrm{F}_{1,2}=0,225 ; \mathrm{F}_{2,21}=0,21 ; \mathrm{P}>0,05\right)$ al igual que las distancias al óptimo $\left(\mathrm{F}_{1,2}=0,049\right.$;

TABLA 2

Diámetro bronquial promedio (d) y su desviación estándar (s) en dos ratas (R1 y R2), clasificados según generación $(\mathrm{G})$, orden de Horstfield $(\mathrm{H})$ y orden de Strahler (S). Entre paréntesis se consigna el número muestral (n)

Average bronchial diameter (d) and standard deviation (s) in two rats (R1 and R2), by generation (G), Horstflied's order $(\mathrm{H})$ and Strahler's order (S). The sample size (n) is showed in brackets

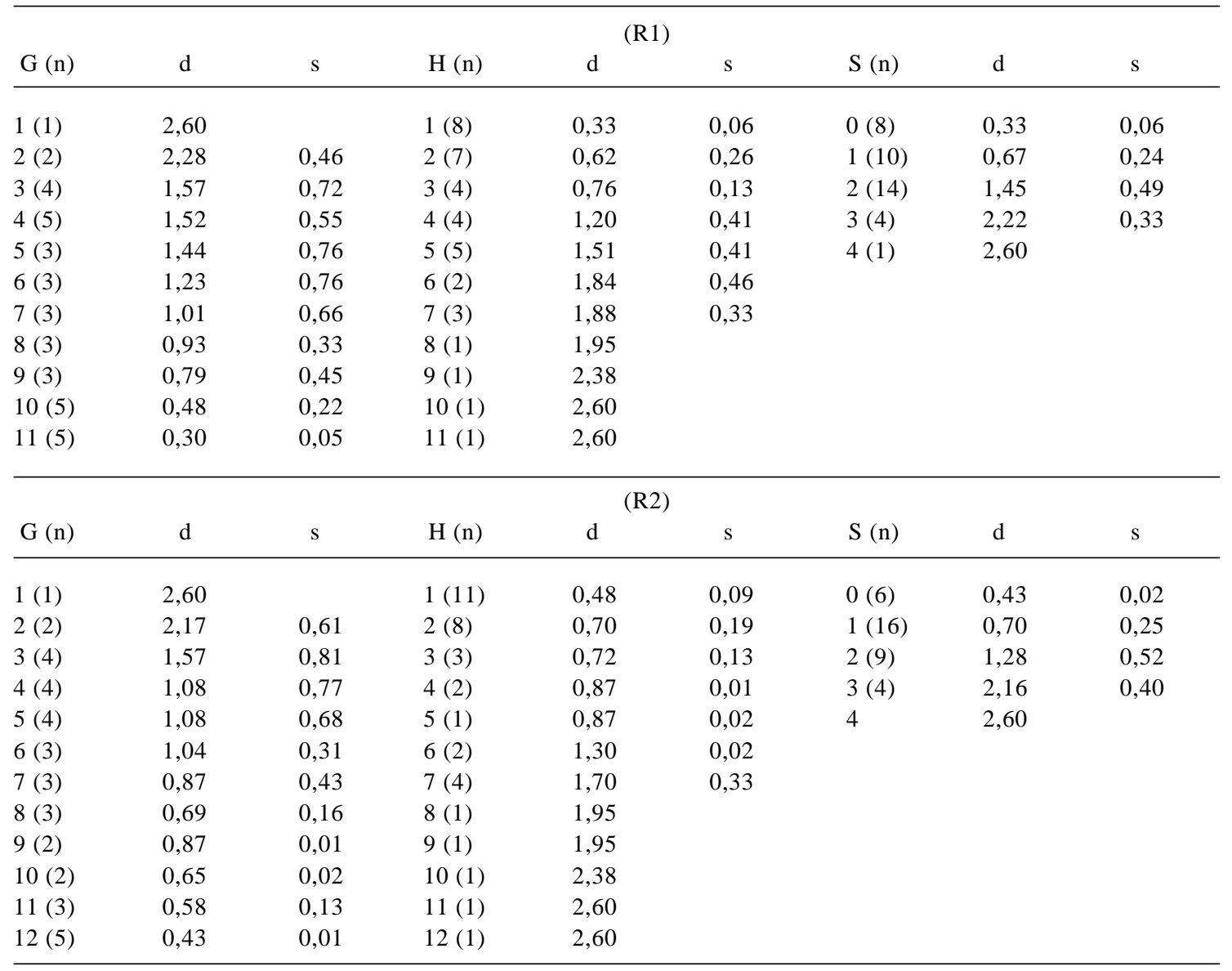


TABLA 3

Bondad de ajuste de los diferentes modelos exponenciales al cambio del diámetro bronquial a lo largo de la vía aérea: d versus G: diámetro-generación; d versus $\mathrm{H}$ : diámetro- ortden de Horstfield; d versus S: diámetro-orden de Strahler y n versus H: número de ramas-orden de

\section{Horstfield}

Goodness of fit of the different models for the variation of the bronchial diameter along the airway: d versus G: diameter-generation; d versus H: diameter-Horstfield's order; d versus S: diameter-Strahler's order and n versus H: number of branches-Horstfield's order

\begin{tabular}{|c|c|c|c|c|c|c|}
\hline Animal & Modelo & Pendiente & Valor de F & $\begin{array}{c}\text { Grados de } \\
\text { libertad }\end{array}$ & Valor de P & Valor de $\mathrm{R}^{2}$ \\
\hline \multirow{4}{*}{$\mathrm{C} 1$} & $\mathrm{~d}$ versus $\mathrm{G}$ & $-0,128$ & 30,2 & 1,43 & $<0,001$ & 41,2 \\
\hline & d versus $H$ & 0,201 & 169,2 & 1,43 & $<0,001$ & 79,7 \\
\hline & $\mathrm{d}$ versus $\mathrm{S}$ & 0,536 & 168,3 & 1,43 & $<0,001$ & 79,7 \\
\hline & $n$ versus $H$ & $-0,311$ & 145,9 & 1,11 & $<0,001$ & 93,0 \\
\hline \multirow{4}{*}{$\mathrm{C} 2$} & $\mathrm{~d}$ versus $\mathrm{G}$ & $-0,178$ & 50,7 & 1,37 & $<0,001$ & 57,8 \\
\hline & $\mathrm{d}$ versus $\mathrm{H}$ & 0,221 & 151,7 & 1,37 & $<0,001$ & 80,4 \\
\hline & d versus $S$ & 0,555 & 121,5 & 1,37 & $<0,001$ & 76,7 \\
\hline & $n$ versus $H$ & $-0,302$ & 99,2 & 1,10 & $<0,001$ & 90,8 \\
\hline \multirow{4}{*}{ R1 } & $\mathrm{d}$ versus $\mathrm{G}$ & $-0,193$ & 66,6 & 1,35 & $<0,001$ & 65,6 \\
\hline & d versus $H$ & 0,242 & 136,5 & 1,35 & $<0,001$ & 79,6 \\
\hline & d versus $S$ & 0,639 & 149,6 & 1,35 & $<0,001$ & 81,0 \\
\hline & $n$ versus $H$ & $-0,363$ & 94,6 & 1,9 & $<0,001$ & 91,3 \\
\hline \multirow{4}{*}{ R2 } & d versus $G$ & $-0,121$ & 31,1 & 1,34 & $<0,001$ & 47,8 \\
\hline & d versus $H$ & 0,173 & 253,1 & 1,34 & $<0,001$ & 88,2 \\
\hline & d versus $S$ & 0,516 & 95,6 & 1,34 & $<0,001$ & 73,8 \\
\hline & $\mathrm{n}$ versus $\mathrm{H}$ & $-0,275$ & 44,5 & 1,10 & $<0,001$ & 81,7 \\
\hline
\end{tabular}

\section{TABLA 4}

Parámetros de la variación exponencial del diámetro bronquial a lo largo de la vía aérea para dos conejos ( $\mathrm{C} 1$ y $\mathrm{C} 2$ ) y dos ratas (R1 y R2). $\mathrm{Rdg}$, $\mathrm{Rd}$ y $\mathrm{Rds}$ corresponden a las razones de variación (incremento o decrecimiento) exponencial del diámetro bronquial en función de la generación, el orden de Horstfield y el orden de Strahler respectivamente. Rb es la razón de incremento exponencial del número de ramas en función del orden de Horstfield. Rd* es el valor esperado suponiendo un flujo óptimo $\left(\mathrm{Rd}^{*}=(\mathrm{Rb})^{1 / 3}\right.$. E $\Delta_{3}$ corresponde al valor esperado para estos parámetros suponiendo un coeficiente de asimetría de $\Delta=3$ (de Horstfield 1990)

Parameters of the exponential variation of the bronchial diameter along the air way in two rabbits (C1 and C2) and two rats (R1 and R2). Rdg, Rd and Rds are the exponential variation rates of the bronchial diameter as function of the generation, the Horstfield's order and the Strahler's order respectively. Rd is the exponential growth rate of the number of branches as function of the Horstfield's order. $\mathrm{Rd}^{*}$ is the expected value assuming an optimal flow into the airway $\left(\mathrm{Rd}^{*}=(\mathrm{Rb})^{1 / 3}\right) . \mathrm{E} \Delta_{3}$ is the expected value for all parameters, assuming an asymmetry coefficient $\Delta=3$ (from Horstflield 1990)

\begin{tabular}{cccccc}
\hline & $\mathrm{Rd}$ & $\mathrm{Rd} *$ & $\mathrm{Rb}$ & $\mathrm{Rdg}$ & $\mathrm{Rds}$ \\
\hline $\mathrm{C} 1$ & 1,223 & 1,109 & 1,364 & 1,136 & 1,709 \\
$\mathrm{C} 2$ & 1,247 & 1,106 & 1,353 & 1,195 & 1,742 \\
$\mathrm{R} 1$ & 1,273 & 1,129 & 1,438 & 1,214 & 1,896 \\
$\mathrm{R} 2$ & 1,189 & 1,096 & 1,316 & 1,129 & 1,675 \\
$\mathrm{E} \Delta_{3}$ & 1,134 & 1,113 & 1,380 & 1,291 & \\
\hline
\end{tabular}


TABLA 5

Ángulos de bifurcación $(\theta)$ y distancias al óptimo para los criterios de mínimos volumen y potencia $\left(\delta_{\mathrm{vp}}\right)$ y mínimos superficie y roce $\left(\delta_{\mathrm{sr}}\right)$ para las bifurcaciones bronquiales en dos conejos ( $\mathrm{C} 1$ y $\mathrm{C} 2)$ y dos ratas (R1 y R2)

Bifurcation angles $(\theta)$ and the distances to the optimum for minimum volume and power $\left(\delta_{\mathrm{vp}}\right)$ and minimum surface and drag $\left(\delta_{\mathrm{sr}}\right)$ criteria for the bronchial junctions in each of two rabbits (C1 and C2) and two rats (R1 and R2)

\begin{tabular}{ccccc}
\hline & $\mathrm{C} 1$ & $\mathrm{C} 2$ & $\mathrm{R} 1$ & $\mathrm{R} 2$ \\
\hline$\theta$ & $63,0 \pm 21,8$ & $65,7 \pm 13,8$ & $58,3 \pm 16,1$ & $65,8 \pm 19,7$ \\
$\delta \mathrm{vp}$ & $0,310 \pm 0,220$ & $0,214 \pm 0,183$ & $0,301 \pm 0,188$ & $0,279 \pm 0,240$ \\
$\delta \mathrm{sr}$ & $0,494 \pm 0,385$ & $0,421 \pm 0,291$ & $0,560 \pm 0,324$ & $0,427 \pm 0,338$ \\
\hline
\end{tabular}

$\left.\mathrm{F}_{2,42}=1,02 ; \mathrm{P}>0,05\right)$, pero las distancias desde una perspectiva de mínimo volumen y potencia fueron menores que desde la perspectiva de mínimos roce y superficie $\left(\mathrm{F}_{1,2}=189,7 ; \mathrm{P}<0,01\right)$ (Tabla 5). La gran mayoría de las bifurcaciones se desvían menos de un $5 \%$ del óptimo esperado desde la perspectiva de mínimo volumen y potencia (sólo un punto se aleja de este límite). En cambio desde la perspectiva de mínimos superficie y roce 6 puntos se encuentran fuera de este rango (Fig. 7).

\section{DISCUSIÓN}

Como era de esperar, los árboles bronquiales analizados presentaron una fuerte asimetría con coeficientes de asimetría de aproximadamente $\Delta=3$, similar a lo encontrado en humanos (Horstfield 1990). Esto se encuentra asociado con la lateralización del corazón y la consiguiente asimetría inducida en los lóbulos pulmonares y en el árbol bronquial. En árboles dicotómicos simétricos el número de ramas $\left(\mathrm{N}_{\mathrm{H}}\right)$ decrece con el orden según $\mathrm{N}_{\mathrm{H}}=2^{\mathrm{G}-1}=2^{\mathrm{Gmax}-\mathrm{H}+1}$ por lo que $\log \mathrm{N}_{\mathrm{H}}=$ cte $-\mathrm{H} \log 2$, de manera que es esperable una razón $\mathrm{Rb}$ $=\operatorname{antilog}(\log 2)=2$. Sin embargo en árboles asimétricos aunque sean dicotómicos, esto no ocurre así porque el número de órdenes y generaciones es diferente en las distintas ramas del árbol (ver Fig. 1). El valor obtenido por nosotros corresponde al esperado construyendo árboles asimétricos de $\Delta=3$ (Horstfield 1990). Como es lógico los diámetros bronquiales decrecieron con la generación y crecieron con el orden, sea este de Horstfield o de Strahler. Los modelos exponenciales fueron adecuados para representar este fenómenos para las generaciones y los órdenes, sin embargo el modelo diámetro-generación mostró siempre un ajuste menor que los modelos diámetro orden. En los primeros, el coeficiente de determinación varió entre 41,2 y $65,6 \%$, en cambio en los últimos este fue siempre superior a 73,8 \%. Además, el ajuste del modelo diámetro-generación difiere significativamente de la curva esperada por Weibel \& Gómez (1962) $(\mathrm{P}<0,05)$ para árboles simétricos en tres de los cuatro casos: C1, C2 y R2 y al comparar con lo esperado para árboles de asimetría $\Delta=3$, se aprecia un ajuste adecuado en $\mathrm{C} 1$ y R2 pero no en $\mathrm{R} 1$ y $\mathrm{C} 2$. Es decir mientras en $\mathrm{C} 1$ y $\mathrm{R} 2$ el desajuste es explicado por la asimetría, en $\mathrm{C} 2$ y R1 no ocurre lo mismo.

Por otra parte en las curvas de decaimiento del diámetro en función de la generación se observa una fluctuación de los puntos alrededor de la recta de regresión que podría ser interpretado como una modulación armónica (oscilación periódica de la escala), sin embargo esta desaparece en los modelos diámetro-orden. De ser real, esta modulación debería mantenerse, pero nuestros resultados refutan tal proposición, coincidiendo con la hipótesis de Horstfield (1990) de que esta es sólo un "artefacto" producido a consecuencia de la asimetría del árbol bronquial, unido al hecho de usar la generación y no el orden de los bronquios. Nuestros resultados apuntan hacia una escala única, muy similar en los cuatro individuos $(\mathrm{Rd})(\mathrm{P}>0,05)$, algo mayores a lo esperado para un árbol asimétrico, sugiriendo aumentos del diámetro con el orden con exponentes (u) entre 0,25 y $0,348\left(\mathrm{~d}=\mathrm{k} 2^{\mathrm{uH}}\right.$, así $\left.\mathrm{u}=\log \mathrm{Rd} / \log 2\right)$. La conocida relación entre flujo y diámetro $\mathrm{q}=\mathrm{kd}^{3} \mathrm{y}$ la dicotomía del árbol bronquial permiten proponer la razón óptima $\mathrm{Rd}^{*}=\mathrm{Rb}^{1 / 3}$, la que también es algo inferior a lo observado en nuestros árboles bronquiales. Sin embargo, el método de Zamir muestra que desde la perspectiva de mínima potencia y volumen prácticamente todas las bifurcaciones se encuentran en una región limitada por una penalización del $5 \%$ del costo, es decir las desviaciones del óptimo son siempre menores a un $5 \%$ con este criterio. Pero en cambio, algunas de las bifurcaciones se alejan de lo óptimo desde 
la perspectiva de la mínima superficie y roce. Esto, unido a lo anterior, podría estar indicando que los bronquios observados no son lo suficientemente finos, pues en estos prevalecería el transporte por difusión, donde el roce y la viscosidad son los factores físicos dominantes. En este sentido Wilson (1967) en un análisis teórico expresa en forma cuantitativa la idea que el diámetro bronquial es limitado en valores pequeños por la creciente resistencia al flujo y en valores grandes por el creciente volumen y espacio muerto. Así la pequeña discrepancia observada entre lo esperado y observado podría ser una limitación del método, sin embargo son necesarios mayores estudios para aclarar este aspecto.

\section{AGRADECIMIENTOS}

Agradecemos la colaboración de Andrés Sazo, quien mantuvo los animales en el vivero y a todo el personal del Servicio de Radiología del Hospital Del Salvador quienes colaboraron en la toma de radiografías. Agradecemos además los valiosos comentarios de los revisores que han ayudado a mejorar este trabajo. Este trabajo fue financiado por el proyecto FONDECYT 1000673 a MCL.

\section{LITERATURA CITADA}

CANALS M, R OLIVARES, F LABRA, L CAPUTO, A RIVERA \& FF NOVOA (1998) Caracterización de la geometría fractal del árbol bronquial en mamíferos. Revista Chilena de Anatomía 16: 237-244.

CANALS M, R OLIVARES, F LABRA \& FF NOVOA (2000) Ontogenetic changes in the fractal geometry of the bronchial tree in Rattus norvegicus. Biological Research 33: 31-35.

DUDLEY R \& C GANS (1991) A critique of symmorphosis and optimality models in physiology. Physiological Zoology 64: 627-637.

DULLEMEIJER P (1998) Optimality in the design of bony elements. En: Weibel ER, CR Taylor \& L Bolis (eds) Principles of animal design: the optimization and symmorphosis debate: 64-70. Cambridge University Press, Cambridge, Inglaterra.

GARLAND T Jr \& RB HUEY (1987) Testing symmorphosis: does structure match functional requirements? Evolution 41: 1404-1409.

GLENNY R \& HT ROBERTSON (1990) Fractal properties of pulmonary blood flow: characterization of spatial heterogeneity. Journal of Applied Physiology 69: 532545.

HORSTFIELD K (1977) Morphology of branching trees related to entropy. Respiration Physiology 29: 179184.

HORSTFIELD K (1990) Diameters, generations and orders of branches in the bronchial tree. Journal of Applied Physiology 68: 457-461.
HORSTFIELD K, FG RELEA \& G CUMMING (1976) Diameter, length and branching ratios in the bronchial tree. Respiration Physiology 26: 351-356.

HORSTFIELD K \& A THURLBECK (1981) Relation between diameter and flow in branches of the bronchial tree. Bulletin of Mathematical Biology 43: 681-691.

JONES JH (1998) Optimization of the mammalian respiratory system: symmorphosis versus single species adaptation. Comparative Biochemistry and Physiology B 120: 125-138.

MANDELBROT BB (1989) Fractal geometry: what is it, and what does it do? En: Fleishmann M, DJ Tildesley $\&$ RC Ball (eds) Fractals in the natural sciences: 3-16. Princeton University Press, Princeton, New Jersey.

McNAMEE JE (1991) Fractal perspectives in pulmonary physiology. Journal of Applied Physiology 71: 1-8.

NELSON TR, BJ WEST \& AL GOLDBERGER (1990) The fractal lung: universal and species-related scaling patterns. Experientia 46: 251-254.

RHORER F (1915) Flow resistance in human air passages and the effect of irregular branching of the bronchial system on the respiratory process in various regions of the lungs. Pfluegers Archives 162: 255-299.

RIGAUT JP (1984) An empirical formulation relating boundary lengths to resolution in specimens showing "non ideally fractal" dimensions. Journal of Microscopy 133: 41-54.

SCHREINER W, F NEUMANN, M NEUMANN, A END \& MR MULLER (1996) Structural quantification and bifurcation symmetry in arterial tree models generated by constrained constructive optimization. Journal of Theoretical Biology 180: 161-174.

SHERMAN TF (1981) On connecting large vessels to small. Journal of General Physiology 78: 431-453.

STRAHLER AN (1957) Quantitative analysis of watershed geomorphology. Transactions of American Geophysical Union 38: 913-920.

WEIBEL ER \& DM GÓMEZ (1962) Architecture of the human lung. Science 137: 577-585.

WEIBEL ER, R TAYLOR \& L BOLIS (1998) Principles of animal design. The optimization and symmorphosis debate. Cambridge University Press, Cambridge, Inglaterra.

WEIBEL ER, R TAYLOR \& H HOPPELER (1991) The concept of symmorphosis: a testable hypothesis of structure function relationship. Proceedings of the National Academy of Sciences USA 88: 10357-10361.

WEIBEL ER, R TAYLOR \& H HOPPELER (1991) Variation in function and design: testing symmorphosis in the respiratory system. Respiration Physiology 60: 325-348.

WEST BJ, V BHARGAWA \& AL GOLDBERGER (1986) Beyond the principle of similitude: renormalization in the bronchial tree. Journal of Applied Physiology 60: 1089-1097.

WILSON T (1967) Design of the bronchial tree. Nature 213: 668-669.

ZAMIR M (1976) Optimality principles in arterial branching. Journal of Theoretical Biology 62: 227 251.

ZAMIR M (1978) Nonsymmetrical bifurcation in arterial branching. Journal of General Physiology 72: 837845. 
ZAMIR M (1986) Cost analysis of arterial branching in the cardiovascular system of man and animals. Journal of Theoretical Biology 120: 111-123.
ZAMIR M \& DC BIGELOW (1984) Cost of de'parture from optimality in arterial Branching. Journal of Theoretical Biology 109: 401-409.

Editor Asociado: F. Bozinovic

Recibido el 1 de octubre de 2001; aceptado el 4 de diciembre de 2001 